\title{
Valores - atributos necesarios para tomar decisiones en salud, a partir de las funciones esenciales de la salud pública
}

\author{
Values - attributes Needed to Make to Decisions in Health, from the Eseential Functions of \\ Public Health
}

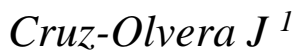

\begin{abstract}
:
Public health functions are understood as the set of actions that must be carried out with the necessary purposes for the achievement of improving the health of the populations. The objective was to analyze the values and attributes such as honesty, knowledge, passion and social sensitivity from the execution of the essential functions of public health in our country, which were decreed by the Pan American Health Organization (PAHO) in 1997. In conclusion, the quality of work based on values, honesty, knowledge, passion and social sensitivity are deficient derived from anti-corruption corruption so that in addition to the execution of essential public health functions in our country is limited, the same quality of poor health.
\end{abstract}

Keywords:

Values-attributes, governmental decisions, corruption, anti-values, essential functions of public health, quality of health care.

\section{Resumen:}

Se entiende como funciones de la salud pública el conjunto de acciones que deben llevarse a cabo con propósitos necesarios para el logro de mejorar la salud de las poblaciones. El objetivo fue analizar los valores y atributos como la honestidad, conocimiento, pasión y sensibilidad social a partir de la ejecución de las funciones esenciales de la salud publica en nuestro país, las cuales fueron decretadas por la organización panamericana de salud (OPS) en 1997. En conclusión, la calidad de trabajo basado en valores, la honestidad, el conocimiento, la pasión y la sensibilidad social son deficientes derivados de la corrupción antivalores por lo que además de la ejecución de las funciones esenciales de salud pública en nuestro país es limitada, por la misma calidad de salud deficiente.

\section{Palabras Clave:}

Valores-atributos, decisiones gubernamentales, corrupción, antivalores, funciones esenciales de la salud pública, calidad de cuidado de la salud.

\section{Introducción}

La salud pública es una ciencia funcional y operativa dentro del sistema de salud en el que las funciones pueden identificar y caracterizar operacionalmente el rendimiento y optimización de sus objetivos con el fin de lograr las once funciones esenciales de la salud pública, las cuales fueron diseñadas por la organización panamericana de la salud en el año 1997, de las cuales se realizara el siguiente análisis crítico.

\section{Análisis crítico}

La primera función esencial de la salud pública se refiere al seguimiento y análisis de la situación sanitaria de la población (Diagnostico situacional), en donde sus principales aspectos hacen referencia a lo siguiente:

Tener una estadística y contar con un sistema de información con acceso en los diferentes niveles de salud del sistema para controlar los cambios en el comportamiento y tomar decisiones basadas en evidencia.

Tener información para evaluar las acciones que demande el sistema de salud y alertas sobre tendencias adversas.

Tener un sistema para detectar, monitorear y evaluar las emergencias en salud pública.

Acceso a la información con suma responsabilidad que demande el sistema de salud. 
La segunda función esencial de la salud pública, hace referencia a la vigilancia, investigación y control de riesgos y daños en salud pública, en donde sus principales aspectos son los siguientes:

Tener un sistema de alerta temprano que permita la detección y control oportunos de brotes y emergencias de enfermedades para salvaguardar el estado de salud de una población.

Toma de decisiones basadas en la mejor evidencia posible.

La tercera función esencial de la salud pública es la promoción a la salud, en donde sus aspectos principales son:

Contar con políticas y programas públicos para fortalecer las habilidades de los ciudadanos, así como de las comunidades favoreciendo todo el tiempo su entorno.

Generación de políticas saludables con fortalecimiento de la participación social y desarrollo de habilidades en las personas que forman parte de la comunidad.

La cuarta función esencial de la salud pública consiste en la participación social y el empoderamiento de los ciudadanos en salud y sus principales aspectos son:

Contar con mecanismos para asegurar la participación de las comunidades en las políticas y programas de salud, así como en la toma de decisiones.

La quinta función esencial de la salud pública, se refiere al desarrollo de políticas, planes y capacidad de gestión y sus principales aspectos son:

Contar con políticas públicas saludables que se traduzcan en normas y programas para desarrollar estilos de vida saludables con elección de las mejores opciones que esto implique promoviendo las condiciones de sostenibilidad para ello.

La sexta función esencial de la salud pública consiste en la regulación y control de la salud pública y sus principales aspectos son:

Contar con un marco legislativo que estandarice y regule las acciones y programas en materia de salud pública en donde se genere ambientes saludables

Ejecución de actividades regulatorias de manera oportuna, consistente y completa; de lo anterior se tiene como base fundamental la Ley General de Salud y la Constitución Política de los Estados Unidos Mexicanos.

La séptima función esencial de la salud pública consiste en la evaluación y promoción del acceso equitativo a los servicios de salud en donde se menciona lo siguiente:

Favorecer la equidad en la distribución de la salud entre la población.

Promoción de la equidad en el acceso a la asistencia sanitaria, servicios, evaluación y promoción con una colaboración de organizaciones no gubernamentales para favorecer a los grupos más vulnerables.

La octava función de la salud pública se refiere al desarrollo de recursos humanos y a la capacitación continua en salud publica en donde se desprende lo siguiente:
Tener una fuerza laboral capacitada con competencias para desarrollar las funciones principales de la salud pública.

Educación, formación y evaluación de la salud pública de manera personal con adherencia a programas de mejora continua con calidad de los servicios.

Definición de requisitos para la licenciatura en profesionales de la salud.

Desarrollo de capacidades para el trabajo interdisciplinario en materia de salud pública.

La novena función de la salud pública se refiere a garantizar la calidad de la salud individual y colectiva: Deberá contar con un sistema de salud que garantice calidad, calidez, eficiencia en la provisión de servicios. Promoción de sistemas permanentes con garantía de calidad

La décima función esencial de la salud pública se refiere a la investigación, desarrollo e implementación de soluciones innovadoras y que deberá contar con lo siguiente:

Investigación relevante y útil para informar la acción que los tomadores de decisiones emprenderán en asuntos relacionas con la salud.

Investigación aplicada para impulsar cambios en la salud pública.

Desarrollo de la propia investigación junto con las autoridades en sus diferentes niveles.

Colaboración interinstitucional para realizar estudios que apoyen la toma de decisiones en salud.

El establecimiento de alianzas con centros de investigación, así como con las instituciones académicas para realizar estudios oportunos no sin antes apoyar la toma de decisiones de la autoridad sanitaria en todo momento con el alcance más amplio de su campo de acción.

\section{Discusión}

En México tener un conocimiento de un sistema de salud que esté preparado para prevenir oportunamente daños causados por desastres con una rehabilitación temprana y adecuada ayudan a tener una reacción en cadena.

Si bien las funciones esenciales podrían significar una serie de indicadores de salud de una población, es fundamental que las funciones esenciales verdaderamente s e cumplan. Son la participación social y el empoderamiento de los ciudadanos en materia de salud los que estrechamente se deben de desarrollar con la responsabilidad compartida en salud pública.

El compromiso y la responsabilidad de mantener una población saludable no debería de ser solo del gobierno, porque es un proceso inmerso en la promoción de la salud en donde los principales valores deberían de ser la honestidad, la competencia, la pasión por el trabajo y lo más importante: la sensibilidad. 


\section{Opinión}

Como en toda empresa, existe una misión y una visión para el mejor otorgamiento de sus servicios, la salud no queda excluida de tal situación, ya que el personal que se encuentra como responsable de una unidad médica independientemente del nivel de atención que se trate, deberá de ofrecer el mejor de los servicios con apego a la misión y visión que se tenga en la institución, sin olvidar que son los valores la pieza fundamental para que se genere un efecto positivo como resultado de los cuales rescato como principales los siguientes: en primer lugar la honestidad, ya que siempre se debe de actuar sin engañar al usuario otorgándole como derecho un acceso a una información clara, oportuna de su padecimiento; así mismo la sencillez es otro valor que agrego como uno de los principales, ya que no por tener un cargo público me convierte en una persona que no sepa escuchar las necesidades que la comunidad demanden cuanto a su salud, por lo cual, siempre se tendrá una apertura para la expresión de las necesidades que demande la comunidad.

\section{Conclusiones}

La calidad del trabajo basada en valores - atributos como la honestidad, conocimiento, pasión y sensibilidad social son estos los que, junto con la ejecución de las funciones esenciales de la salud pública, elevaran la calidad en la eficiencia de la salud en el país.

Cabe señalar que actualmente el valor de la equidad es una situación que durante los últimos años ha tomado gran relevancia de manera social, pues no debe hacerse distinción para el acceso a la salud de las personas independientemente de su género, ideología política, religiosa, cultural o incluso de preferencia sexual; y lo rescato como piedra angular para garantizar el pleno nivel de salud en los individuos y que está plasmado como un derecho constitucional.

En cuanto a las funciones de la salud pública es importante que antes de ofrecer servicios de salud, tengamos un conocimiento sobre el diagnostico situacional de esa población, en donde conozcamos sus necesidades pero con evidencias que sustenten el desarrollo de políticas y programas para el aterrizaje de los recursos que serán empleados en favor de los ciudadanos donde lo fundamental será conocer las principales causas de morbi -mortalidad de la comunidad, así como grupos poblacionales catalogados como de riesgo para los cuales estarán enfocados los principales programas de salud que se demanden.

De tal manera, la salud pública ha mencionado como una de sus principales funciones el tener capacitado a todo su personal el cual no podrá ofrecer salud sin un conocimiento claro el cual deberá de actualizarse continuamente, ya que ante un mundo globalizado no puede haber rezago en materia de capacitación.

Finalmente, rescato como una de las principales funciones es de la salud pública sobre la creación de políticas y programas de salud aterrizados según las necesidades dela comunidad lo cual se hace con evidencia de lo ya vivido con experiencias anteriores, por lo que se proyectan el financiamiento de los recursos para atender las emergencias en salud pública y necesidades básicas que demande la comunidad.

\section{Referencia}

(1) Hernández Medina Miriam Selene, Hernández Serrano Carolina, López González Ana Laura, Barragán López Norma, Islas Vega Irasema, Mariela Lizbeth Cobián Díaz, Reynoso Vázquez Josefina, Ruvalcaba Ledezma Jesús Carlos. Values-attributes Needed to Make Decisions in Health, from the Essential Functions of Public Health. American Journal of Public Health Research, vol. 5 (5): 154-158 